\title{
Le choix du prénom. Des régularités statistiques aux mécanismes cognitifs
}

Jean-Michel Berthelot

\section{OpenEdition}

\section{Journals}

Édition électronique

URL : http://journals.openedition.org/ress/354

DOI : $10.4000 /$ ress.354

ISSN : 1663-4446

Éditeur

Librairie Droz

Édition imprimée

Date de publication : 1 mars 2004

Pagination : 13-21

ISBN : 2-600-00941-8

ISSN : 0048-8046

\section{Référence électronique}

Jean-Michel Berthelot, « Le choix du prénom. Des régularités statistiques aux mécanismes cognitifs », Revue européenne des sciences sociales [En ligne], XLII-129 | 2004, mis en ligne le 04 novembre 2009, consulté le 06 mai 2019. URL : http://journals.openedition.org/ress/354 ; DOI : 10.4000/ress.354 
Jean-Michel BERTHELOT

\section{LE CHOIX DU PRÉNOM. DES RÉGULARITÉS STATISTIQUES AUX MÉCANISMES COGNITIFS}

Philippe Besnard se félicitait d'avoir écrit l'un des best-sellers de la sociologie française $^{1}$. À bon droit. Ironiquement, il savait que la majeure partie des sociologues l'ignorait, si ce n'est peut-être ceux qui, un jour de désarroi, avaient acheté le Besnard-Desplanque avant d'affronter le Laurence Pernoud.

La méconnaissance est encouragée par le livre qui annonce la cote des prénoms et permet à chacun, au prix de quelques calculs élémentaires, de savoir si son choix (ou celui de ses parents), fut innovant, classique ou conformiste. Belle entreprise de démystification des légendes familiales qui nous apprend que, née entre 1950 et 1960, se prénommer Martine est d'une banalité confondante, tandis que choisir Charles pour l'héritier de la famille en 1980, ne fait que confirmer un retour du prénom depuis le début des années soixante-dix.

La méconnaissance n'est pas seulement injuste. Elle est préjudiciable. Les quatre-vingts pages qui précèdent le catalogue des prénoms constituent un modèle d'approche durkheimienne et une source d'inspiration particulièrement originale pour la philosophie des sciences sociales. Le problème peut être résumé en deux phrases. Nous avons tous d'excellentes raisons de choisir tel prénom pour notre enfant; ce sont même des raisons strictement privées (il sonne bien avec le nom de famille; il nous rappelle un personnage littéraire; il est associé à un moment de l'histoire du couple ou de l'un de ses membres etc.). Simultanément, «pour chaque sexe, à un moment donné, les dix prénoms les plus fréquents suffisent à désigner un 1/3 des nouveau-nés » et, «aucun de ces prénoms les plus courants ne se retrouve vingt ans plus tard au palmarès des 10 premiers » $\left(\mathrm{p} .9^{2}\right)$

1.

La structure de l'énigme est donc du même type que celle du Suicide: un phénomène qui ne semble relever que de décisions individuelles et dont on attendrait une dispersion aléatoire, manifeste des régularités particulièrement fortes. La méthodologie est, également, durkheimienne: divers échantillons couvrant un peu plus de 120 ans et comptant environ 2600000 individus, permettent de mesurer les fréquences d'apparition des prénoms et de les croiser avec quelques

Ph. Besnard et G. Desplanques, Un prénom pour toujours. La cote des prénoms hier, aujourd' hui et demain, Paris, Balland, 1986.

$2 \quad$ La pagination que je fournis est celle de l'édition de 1988. 
caractéristiques sociales fondamentales. Le modèle explicatif, en revanche, est individualiste: le choix du prénom est un phénomène de mode, d'agrégation de choix individuels pris entre les deux caractéristiques des phénomènes de mode: la distinction et le conformisme.

Simultanément, Philippe Besnard met le doigt sur l'originalité du prénom et l'intérêt spécifique de son étude: «Le prénom est le bien de mode par excellence (...) (C'est) un bien gratuit dont la consommation est obligatoire (...) Voilà ce qui démarque le choix des prénoms de tout autre acte de consommation et en fait le terrain de manœuvre privilégié du phénomène de mode dans ce qu'il a de purement social». (p. 11)

D'une certaine façon, il s'agit d'un phénomène qui se donne immédiatement «épuré »: aucune détermination externe, utilitaire, symbolique ou de coût, ne pèse sur le choix du prénom.

Les résultats empiriques confortent le choix du modèle. Cependant, et c'est la thèse que je développerai, ils posent la question suivante: comment faire coexister, dans un même modèle explicatif, les régularités statistiques établies et les raisons - ou les croyances - des acteurs? Cette question n'est pas très originale pour qui est familier de la tradition weberienne. Elle trouve cependant, sur ce terrain, des possibilités inédites d'approfondissement, voire de mise à l'épreuve.

2.

On peut résumer l'analyse menée par Philippe Besnard en l'organisant autour de deux pôles: les caractéristiques sociales des prénoms; les facteurs sociaux du choix. Je serai bref, renvoyant les lecteurs au texte pour davantage de détails.

\section{Caractéristiques sociales des prénoms}

a. Le répertoire global des prénoms utilisé par la population française depuis le XVI ${ }^{\mathrm{e}}$ siècle est de plus de 2000 (les prénoms en usage dans les communautés récemment immigrées ne sont pas pris en compte dans l'enquête). À un moment donné, les prénoms en usage constituent un sous-ensemble de ce répertoire. Ce stock lui-même tend à croître et à se renouveler de plus en plus rapidement (p. 29).

b. Les prénoms ont un parcours déterminé:

- Celui-ci oscille entre une trajectoire modale que l'on peut décrire en 5 périodes clairement différenciées (de l'émergence au déclin) et une trajectoire classique quasi parallèle à l'échelle du temps (p. 33-35).

- La durée de vie d'un prénom est d'environ 40 ans, dont 20 ans de réelle visibilité (évaluée à une fréquence supérieure à un seuil de 1\%). Lorsqu'un prénom adopte une trajectoire modale, il connaît en moyenne une phase d'émergence de 9 ans, une phase d'ascension de 5 ans, une phase conformiste de 6 à 7 ans, suivie d'un déclin de 8 ans et d'une agonie de 10 (p. 35-36).

- Le retour d'un prénom tombé en désuétude s'opère à l'échelle séculaire: un siècle et demi séparent en général les pics de plus forte fréquence (Julie, 1830/1980; Pauline, 1850/1990...) 
- Si un prénom a été brillant (sommet élevé), s'il a été à la mode (trajectoire modale), et si sa carrière date de 40 à 90 ans, alors il a d'autant moins de chance d'être choisi (p. 39). Cette quasi-loi sous-tend les choix: «L'allure de la courbe de diffusion passée, combinée à la distance qui nous en sépare est, en matière de prénoms comme en beaucoup d'autres, la clé principale de nos goûts et dégoûts du moment» (p. 39)

c. La co-occurrence des prénoms à un moment donné, le remplacement de certains prénoms par d'autres, semblent associés à des règles «d'affinité sonore, qui rassemble les prénoms en vogue à un moment donné » (p. 40). Par exemple:

- terminaisons communes: vague des -ette (Paulette, Georgette...) entre 1920 et 1940, puis des -iane (Josianne, Vivianne...), des -ine, des -ie, des -ia ou -a...

- radical ou première syllabe, voire consonne, communs: Christian $\rightarrow$ Christophe; Michel $\rightarrow$ Michael; Céline et Cécile; les «Quatre J» Jessifer, Jessica, Jonathan, Jérémy...

\section{Facteurs sociaux du choix}

Le choix des prénoms varie selon les groupes sociaux, le lieu d'habitation, l'âge des parents ou le rang dans la fratrie. Les corrélations, que peuvent fournir les données, invitent cependant à des affirmations nuancées.

a. Il y a un parcours social des prénoms, qui grosso modo se propagent du haut au bas de l'échelle sociale; les animaux sont parfois de la partie comme terrain d'expérimentation (Ulysse, Pénélope...) (p. $52^{3}$ ). Cela suggère un modèle de diffusion et d'innovation à deux dimensions, verticale (place dans l'échelle sociale), horizontale (degré de sociabilité et d'échanges): «Un prénom - ou n'importe quel bien de mode - se propage aussi comme une maladie contagieuse. Nos chances d'être touchés par l'épidémie sont fonction des occasions que nous avons de l'approcher et donc de la fréquence de nos contacts avec les autres » (p. 54)

b. On peut vouloir distinguer un goût bourgeois d'un goût populaire. Il y faut d'abord des précautions méthodologiques: un prénom bourgeois n'est pas un prénom qui sonne bourgeois, mais un prénom choisi de façon plus fréquente par les cadres. Il peut en revanche, compte tenu de la différence d'effectifs des divers groupes sociaux être davantage répandu dans les couches moyennes ou populaires. L'enquête révèle qu'il s'agit le plus souvent de prénoms stables, classiques, plus rares. Mais l'hypothèse d'une distinction fondée sur la rareté et le rejet de la mode n'est pas validée: certains prénoms rares ne sont pas bourgeois (Lydie, Nadège, Nadia...); d'autres ont pu atteindre les sommets de

«Il n'y a pas lieu d'alerter la SPA, bien au contraire. Nos animaux familiers sont autrement choyés que ne l'étaient les bonnes. Tandis que la domesticité ramassait les miettes du grand festin de la mode, les chats et les chiens d'aujourd'hui, qui dédaignent nos restes, nous regardent du haut de la pyramide sociale de l'innovation». 
la mode, tout en le restant (Philippe, Olivier, Arnaud...). Le goût populaire se traduit pour sa part par un recours nettement plus fréquent à des prénoms nouveaux, d'importation anglo-saxonne, notamment (Anthony, Gregory, Kevin...)

En lien avec les facteurs sociaux positionnels, d'autres influences, d'ordre culturel, peuvent intervenir dans le choix du prénom: la tradition religieuse, l'imitation des vedettes du spectacle et autres héros de films ou de séries télévisées. Là encore, le verdict des faits invite à la prudence.

a. Les influences religieuses sont ambiguës : on peut constater des liens entre des canonisations et l'engouement pour certains prénoms (Thérèse, Bernadette). Cet effet a également ses formes locales, dans la préférence pour un prénom associé à une sainte particulière (Colette en France-Comté, Geneviève dans la région parisienne). Mais toute esquisse de généralisation se heurte à autant de contre-exemples.

b. Le rôle des media paraît évident au sens commun et à l'expérience ordinaire: la floraison des Brigitte, des Sylvie, des Thierry dans les années soixante évoque irrésistiblement Brigitte Bardot, Sylvie Vartan, Thierry la Fronde... Chaque décennie a ainsi ses stars dont le rayonnement semble répandre le prénom. Les faits ne confirment pas cette apparence:

- La personnalité la plus forte est impuissante à interrompre la chute ou la disparition cyclique d'un prénom: Charles décline régulièrement depuis le début du siècle, indépendamment de la Résistance et de la 5 ème république. Bien que l'amorce de son retour, à partir du début des années soixante-dix, pourrait être interprétée comme une influence posthume du Général de Gaulle, elle correspond de fait au retour cyclique du prénom.

- Il y a souvent coïncidence entre le pic d'un prénom et la notoriété des vedettes. Or, la relation doit être lue, la plupart du temps à l'inverse: ce sont les vedettes qui choisissent un pseudonyme à la mode ou en train de le devenir: Alain (Delon), Julien (Clerc), Michèle (Morgan)...

- On peut peut-être repérer l'effet plus spécifique de certaines chansons à la mode, contribuant à lancer un prénom: Cecile, Mélissa, Nathalie... Dans deux cas, au moins, le mouvement d'amplification est flagrant (Laeticia, 1964, Gainsbourg); Cécile (1962, Nogaro). Mais il s’agit néanmoins de prénoms en phase d'émergence.

Les faits sont donc clairement établis. D'autres sans doute manquent, permettant divers affinements. Mais ceux-ci, sauf à soupçonner des erreurs de traitement statistique, sont robustes. Ils échappent même, grâce à la spécificité des prénoms, aux objections habituelles sur l'arbitraire des catégorisations ou l'incertitude des enregistrements. Les objections que J. D. Douglas adressaient aux statistiques du Suicide - et que Philippe Besnard connaissait bien -, sont ici sans prise: si l'on peut suspecter les familles, lors des déclarations mortuaires, de vouloir éviter l'opprobre du suicide, les prénoms eux, sont officiellement enregistrés. Les biais, tenant notamment aux prénoms multiples, non pris en compte dans l'enquête, sont minimes. La question est donc: comment expliquer ces faits? 
3.

Le souci de Philippe Besnard, dans le texte précédant la cote des prénoms, semble être d'abord descriptif et critique: descriptif en ce qu'il s'agit de cerner la nature sociale des prénoms; critique, en ce qu'il s'agit de tordre le cou à quelques idées fausses. Le modèle explicatif est donc peu approfondi, même s'il est posé avec fermeté:

a. Le choix du prénom est affaire de mode;

b. La mode fonctionne selon les deux dimensions de la distinction et du conformisme.

c. Rien d'autre, pourrait-on ajouter, n'est nécessaire pour comprendre le jeu de nos goûts et dégoûts.

Ce modèle permet même de construire une explication probabiliste du choix et de la confronter à des prévisions.

a. Un prénom a d'autant moins de chance d'être choisi qu'il a été à la mode, a atteint des sommets et a été en vogue dans une période antérieure de 40 à 90 ans (cf. supra)

b. La nature innovante, conformiste ou démodée du choix d'un prénom dépend du moment de son parcours, pondéré par les caractéristiques sociales des parents: l'appartenance à un milieu de cadres, à Paris, pour une femme jeune, ayant un premier enfant, prédispose à l'innovation, alors que le statut d'agriculteur, à la campagne, pour une femme plus âgée et un deuxième ou troisième enfant, prédispose au conformisme (p. 95-97).

Restent en suspens, cependant, diverses questions :

1. Les bonne raisons des acteurs sont-elles de fausses raisons? Apparemment non, puisque ces raisons sont compatibles avec les choix et portent sur ce que peuvent maîtriser les acteurs : choisir Julien, en 1980, peut être justifié par une passion littéraire pour le Rouge et le Noir ou par le souvenir ému de Julien Clerc dans Hair, tout en étant un choix innovant. Le moment où en est Julien dans sa carrière de prénom n'invalide pas les raisons personnelles que j'ai de le choisir. En revanche, mes raisons ne peuvent en rien expliquer, qu'au même moment, un nombre indéterminé de parents aient d'excellentes raisons - identiques ou différentes - de choisir Julien et de contribuer ainsi, en toute ignorance, à son ascension. Julien étant un prénom et non un bien de consommation, aucune utilité n'explique la rencontre de nos choix.

2. Les bonnes raisons sont-elles l'expression de mécanismes causaux? La difficulté est la suivante: comment des caractéristiques ignorées des acteurs (situation des divers prénoms dans leur parcours social) peuvent-elles influer sur leur choix et prendre l'apparence de raisons intimes? Comment saisir le parcours qui mène d'une régularité non disponible à un choix intentionnel qui la conforte de façon non intentionnelle? 
3. De quelle nature est le mécanisme causal? Tient-il à des propriétés inhérentes aux prénoms? Mais ces propriétés sont phonologiques (cf. les terminaisons ou les radicaux communs) et statistiques (fréquence différentielle). Si propriétés sémantiques il y a, elles sont beaucoup plus floues: sait-on vraiment, en prénommant sa fille «Bénédicte», que l'on fait un choix «bourgeois »? Penset-on aux lignées royales lorsque l'on opte pour Louis ou Edouard? En fait, toutes ces propriétés sont relationnelles: elles n'existent, pour un prénom donné, que dans une relation avec les autres prénoms (euphonique, statistique) ou avec des caractéristiques sociales et historiques contingentes.

Les modèles explicatifs d'une influence traditionnelle ou positionnelle (reproduction et distinction sociale) s'avérant tautologiques, deux hypothèses semblent prédominer: celle du phénomène de mode et celle des bonnes raisons. Le problème est que, même en les poussant, on ne parvient pas à avancer dans les questions précédentes:

- le modèle de la mode (diffusion/distinction) est explicatif au sens de Hempel. Comme nous l'avons vu plus haut, il produit une loi de probabilité permettant, non d'anticiper un choix précis, mais de séparer assez bien le corps des prétendants légitimes de celui des recalés définitifs. Il permet également de faire des pronostics assez précis sur le devenir des prénoms. Simultanément, il ne répond pas aux prérequis d'une explication compréhensive: les processus intentionnels et les mécanismes cognitifs par lesquels les choix se font et, in fine, les carrières des prénoms se poursuivent, restent totalement opaques.

- le modèle du choix rationnel (ou des bonnes raisons) peut être présenté de la façon suivante. L'agent est face à un répertoire de possibles déterminé (environ 2000 prénoms). Ce répertoire se ramène, à un instant donné, à un stock effectivement en usage (cf. supra). L'agent va puiser dans ce stock en fonction de préférences relevant de considérations multiples: projections identitaires ( «je ne vois pas mon fils avec ce prénom!»), esthétique («ça sonne bien »), biographiques («c'est la chanson de mon enfance, mon film culte, l'hérö̈ne absolue...»), émotionnelles («ça rendra les copines folles de jalousie!»), politiques («cela fera plaisir à la belle famille!») etc. Tout ceci délimite un sous-stock, sans doute beaucoup plus restreint. L'intérêt du modèle est de fournir des mécanismes de sélection susceptibles de sous-tendre de façon acceptable les raisons avancées des agents. Le problème est que ce type de considération (puisqu'il n'y a pas de valeur ou de propriété objective de référence) devrait aboutir à la plus grande dispersion dans une relative concentration: la plus grande dispersion, parce que l'on ne voit pas quels mécanismes pourraient la réduire; dans une relative concentration, car rien, dans ce modèle, ne peut engendrer un véritable renouvellement du stock de départ ${ }^{4}$. Dans les deux cas, on aboutit à une situation différente des faits.

Ces deux points mériteraient sans doute d'être davantage argumentés. On peut objecter au premier que l'espace de choix est réduit par celui de la sociabilité (on connaît les prénoms de son environnement relationnel), et au second qu'un stock s'use naturellement et contraint au renouvellement. Mais précisément, pour le premier, le souci de distinction peut amener à sortir du répertoire immédiat, tout en restant dans le stock en usage et, pour le 
Nous nous trouvons donc dans la situation, fréquente en sciences sociales, où deux modèles explicatifs différents semblent apporter une explication complémentaire, sans que l'on soit capable de reconstituer les liaisons causales manquantes.

Je ne prétendrai pas sortir la solution, comme le lapin du chapeau. Seulement, je voudrais, pour finir, esquisser les formes possibles d'un modèle susceptible d'être, au moins indirectement, mis à l'épreuve.

4.

J'adopterai les propositions suivantes :

A.

1) Les prénoms disponibles à un moment donné constituent un stock dont on peut mettre à jour des mécanismes de composition (formation linguistique, origine culturelle, diffusion), de distribution, et de trajectoires (il s'agit de l'ensemble des faits établis par Philippe Besnard).

2) Ce stock est géré par des choix individuels qui s'exercent selon des préférences personnelles, constitutives des bonnes raisons de préférer tel prénom à tel autre, ou d'hésiter entre tel ou tel prénom.

3) Ces deux mécanismes expliquent des faits différents: rien, rationnellement, en 2 ne permet d'expliquer la dispersion faible du stock global et le point atteint dans la carrière par chaque prénom; rien en 1 , ne permet de comprendre comment ces mécanismes peuvent résulter des raisons des acteurs.

B

1) On fera l'hypothèse qu'entre l'état objectif du stock au moment t et son état au moment $\mathrm{t}+1$, l'agrégation des décisions individuelles aboutissant au devenir du stock selon des régularités prévisibles, implique divers phénomènes cognitifs distincts :

a) Un phénomène de rétention et de gestion de l'information: nous retenons mieux certains prénoms (ou certaines séries de prénoms) que d'autres. Ceci peut sembler lié à leur plus grande fréquence d'apparition dans notre environnement, aux expériences personnelles auxquelles ils sont associés (lecture, cinéma, rencontres amoureuses etc.) mais aussi à leur structure phonique et aux associations esthétiques et sémantiques qu'elle peut suggérer. En résumé, le sous-ensemble personnel du stock global que nous avons en mémoire dépend des facteurs suivants:

i) Notre espace de sociabilité

ii) L'intensité des expériences vécues (engendrant goûts et dégoûts)

second, les prénoms ne sont ni des marchandises ni des biens culturels. Un épuisement «mécanique» n'est que l'effet cumulé de changements de choix. 
iii) Les modalités de mémorisation et de stockage de l'information, liées notamment à sa structure phonique

iv) L'évolution du stock global, qui renforce la présence de certains prénoms - et de certaines chaînes phoniques - dans l'environnement, et en laisse s'estomper d'autres.

Ce stock serait un stock en mémoire vive, immédiatement disponible, marqué par la fréquence de ses éléments dans l'environnement vécu et la force des affects qui peuvent leur être associés. Sa présence et sa forme différentielle pourrait être aisément testée par un protocole de questionnement et de commentaires auprès d'une population déterminée.

b) Un phénomène de recherche d'information: les prénoms qui viennent spontanément à l'esprit peuvent ne pas satisfaire les parents. Ils peuvent vouloir innover ou retrouver des prénoms anciens. Jouent ici le mécanisme global de distinction (se distinguer différentiellement des proches) et la capacité d'investigation et d'expertise que recèlent les compétences sociales des parents (prénoms littéraires, historiques, traditionnels, médiatiques...)

c) Un phénomène de justification du choix ultime, qui associe sans doute, sur la base du stock restreint ainsi constitué, désir de distinction et de conformité, compromis entre les parents, voire entre les parents et les enfants ou les parents et la famille. Les jugements évaluatifs ordinaires qu'occasionne en général le choix d'un prénom (y compris pour les autres), sont de bons guides: «Il sonne mal»; «On ne voit que ce prénom en ce moment»; «C'est démodé»; «C'est difficile à porter»... Ils explicitent bien la perception de l'espace distinction/conformité au sein duquel les acteurs choisissent les prénoms que les mécanismes cognitifs de tri, et les opérations conscientes de recherche, ont sélectionnés.

Résolvons nous les questions: non. Mais nous avançons sur la voie tendant à saisir comment les propriétés sociales des prénoms sont investies par des mécanismes cognitifs susceptibles de les reproduire.

Ces propriétés sont relationnelles et partiellement opaques aux agents: les propriétés sémantiques (associées à des événements et des personnages plus ou moins actuels ${ }^{5}$ ) sont floues, ainsi que les propriétés euphoniques ${ }^{6}$; les propriétés statistiques, qui relèvent de fréquences différentielles, sont opaques. Cependant quelque chose de ces propriétés est perceptible aux agents et reçu comme information. Il n'est pas déraisonnable de penser que l'expérience informationnelle de vingt ou trente ans, qui, chez un agent, précède la paternité ou la maternité, enregistre, par la seule accumulation des occurrences, certaines tendances: des prénoms qui reviennent de plus en plus dans l'environnement, ou au contraire

Quel jeune, aujourd'hui, associera Maurice à Maurice Chevalier, ou Philippe à Philippe Pétaint ou Jacky à Mme Kennedy?

${ }^{6}$ Suis-je conscient de la vague des -iane, lorsque je prénomme ma fille Josianne ou Sylvianne? 
s'éclipsent; des prénoms faiblement usités, mais d'une constance discrète; des prénoms littéraires qui exercent leur charme secret; des prénoms médiatiques qui semblent brusquement tout envahir. Il n'est pas déraisonnable de penser non plus que les processus de mémorisation, d'investissement affectif, de pertinence esthétique qui, pour chaque agent, à partir du stock commun, construisent son choix, ont la capacité de reproduire, par agrégation, les tendances existantes. En effet, le même mécanisme est à l'œuvre, mais saisi à deux échelles différentes: un mécanisme de distinction, fondé chez les parents sur de bonnes raisons identitaires (que leur enfant s'appelle Aliénor ou Sabrina) et entraînant pour le stock son renouvellement; un mécanisme de conformisme, fondé chez les parents sur le souci de ne pas exclure leur enfant de leur communauté d'appartenance, et perceptible, au niveau agrégé dans l'absence de ruptures fortes des trajectoires et dans leur développement sur un espace temporel large.

Nous retrouvons, évidemment, l'opposition entre distinction et conformisme, mais appuyé sur des mécanismes cognitifs dont l'approfondissement mérite d'être entrepris.

Université de Paris IV-Sorbonne

jean-michel.berthelot@paris4.sorbonne.fr 